\title{
The product formula in cohomological Hall algebras
}

\author{
Xinli Xiao \\ Mathematical Department, Kansas State University, Cardwell Hall 128, Manhattan, Kansas, 66502 \\ E-mail address: xiaoxl@math.ksu.edu
}

\begin{abstract}
This is an expository article. It gives a detailed explanation of the product formula of Cohomological Hall algebra.
\end{abstract}

\section{Introduction}

In [3], Maxim Kontsevich and Yan Soibelman introduced a remarkable algebra called Cohomological Hall algebra, which has a very complicated product formula. This expository article is devoted to explain this product formula in details.

The paper is divided into three parts. In Section 2, the definition of Cohomological Hall algebra and the definition of its product is recalled. Since these things are highly related to equivariant cohomology, in Section 3, a quick access to important results of equivariant cohomology we need in this paper are given. At last, using these results, the formula is explained in details in Section 4.

\section{The definition of Cohomological Hall algebra}

2.1. Quiver moduli stack. Let $Q$ be a quiver, $I$ be the set of its vertices and $H$ be the set of arrows. For a fixed dimension vector $\gamma=\left(\gamma^{i}\right)_{i \in I}$, we have the representation space $M_{\gamma}=\bigoplus_{\alpha: i \mapsto j} \operatorname{Hom}\left(\mathbb{C}^{\gamma^{i}}, \mathbb{C}^{\gamma^{j}}\right)$ and the group $G_{\gamma}=\prod_{i \in I} G L_{\gamma^{i}}(\mathbb{C})$ acting on it. The action is defined by conjugation:

$$
g \cdot f=\left(g_{j} f_{i j} g_{i}^{-1}\right)_{\alpha: i \mapsto j},
$$

for $g=\left(g_{i}\right)_{i \in I} \in G_{\gamma}$ and $f=\left(f_{i j}\right)_{\alpha: i \mapsto j} \in M_{\gamma}$. Since $M_{\gamma}$ can be treated as the space of representations of $Q$ in coordinate spaces of dimensions $\gamma$, and $G_{\gamma}$ is the automorphism group of the isomorphism classes of representations, the quotient stack $\left[M_{\gamma} / G_{\gamma}\right]$ is the stack of representations of $Q$ with dimension vector $\gamma$. 
2.2. Correspondence and pullback-pushforward construction. Fix any two dimension vectors $\gamma_{1}$ and $\gamma_{2}$ and denote $\gamma=\gamma_{1}+\gamma_{2}$. Denote by $M_{\gamma_{1}, \gamma_{2}}$ the space of representations of $Q$ in coordinate spaces of dimensions $\left(\gamma_{1}^{i}+\gamma_{2}^{i}\right)_{i \in I}$ such that the standard coordinate subspaces of dimensions $\left(\gamma_{1}^{i}\right)_{i \in I}$ form a subrepresentation. Obviously $M_{\gamma_{1}, \gamma_{2}}$ is an affine space, and also a closed subspace of $M_{\gamma}$. The group $G_{\gamma_{1}, \gamma_{2}} \subset G_{\gamma}$ consisting of transformations preserving subspaces $\mathbb{C}^{\gamma_{1}^{i}} \subset \mathbb{C}^{\gamma^{i}}$ for all $i \in I$, acts on $M_{\gamma_{1}, \gamma_{2}}$. The quotient stack $\left[M_{\gamma_{1}, \gamma_{2}} / G_{\gamma_{1}, \gamma_{2}}\right]$ is the correspondence we are looking for.

Now let's apply the standard pullback-pushforward construction to these objects. Since $\left[M_{\gamma_{1}, \gamma_{2}} / G_{\gamma_{1}, \gamma_{2}}\right]$ contains information about $\left[M_{\gamma_{1}} / G_{\gamma_{1}}\right],\left[M_{\gamma_{2}} / G_{\gamma_{2}}\right]$ and $\left[M_{\gamma} / G_{\gamma}\right]$, there are three natural projections:

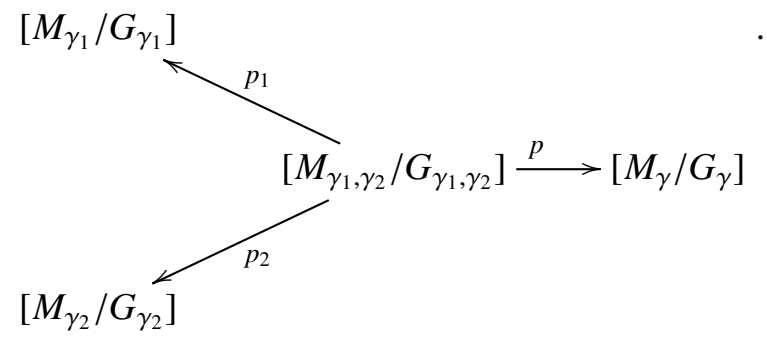

Note that $p$ is a proper morphism of smooth Artin stacks. Hence it induces the pushforward map on cohomology. Combining it with the pullback $\left[M_{\gamma_{1}} / G_{\gamma_{1}}\right] \times$ $\left[M_{\gamma_{2}} / G_{\gamma_{2}}\right] \leftarrow\left[M_{\gamma_{1}, \gamma_{2}} / G_{\gamma_{1}, \gamma_{2}}\right]$, we obtain

$m_{\gamma_{1}, \gamma_{2}}: H^{*}\left(M_{\gamma_{1}} / G_{\gamma_{1}}\right) \otimes H^{*}\left(M_{\gamma_{2}} / G_{\gamma_{2}}\right) \stackrel{p_{1}^{*} \otimes p_{2}^{*}}{\longrightarrow} H^{*}\left(M_{\gamma_{1}, \gamma_{2}} / G_{\gamma_{1}, \gamma_{2}}\right) \stackrel{p_{*}}{\longrightarrow} H^{*}\left(M_{\gamma} / G_{\gamma}\right)$.

2.3. The cohomology. From [2], the cohomology of quotient stacks can be realized as the equivariant cohomology of the underlying space on which the gauge group acts. Then the above map (2.3) can be modified to equivariant cohomology:

$$
m_{\gamma_{1}, \gamma_{2}}: H_{G_{\gamma_{1}}}^{*}\left(M_{\gamma_{1}}\right) \otimes H_{G_{\gamma_{2}}}^{*}\left(M_{\gamma_{2}}\right) \stackrel{p_{1}^{*} \otimes p_{2}^{*}}{\longrightarrow} H_{G_{\gamma_{1}, \gamma_{2}}}^{*}\left(M_{\gamma_{1}, \gamma_{2}}\right) \stackrel{p_{*}}{\longrightarrow} H_{G_{\gamma}}^{*}\left(M_{\gamma}\right) .
$$

Definition-Proposition 2.1. [3] Let $H_{\gamma}=H_{G_{\gamma}}^{*}\left(M_{\gamma}\right)$, and $H_{Q}=\oplus_{\gamma} H_{\gamma}$. Equipped it with a multiplication $m$ whose restriction over $H_{\gamma_{1}} \otimes H_{\gamma_{2}}$ is $m_{\gamma_{1}, \gamma_{2}} . m$ is associative. $H_{Q}$ is called the Cohomological Hall algebra associated to the quiver $Q$.

\section{Preliminaries}

3.1. Notations. Let $G$ be a compact, connected Lie group of rank $n$ and $T$ a maximal torus in $G$. A character of the torus $T$ is a multiplicative Lie group 
homomorphism of $T$ into $\mathbb{C}^{*}$. Fix a character $\lambda$, we can construct a 1-dimensional representation $\mathbb{C}_{\lambda}$ of $T$ via $t \cdot \mu=\lambda(t) \mu$ for $t \in T$ and $\mu \in \mathbb{C}$. On the Lie algebra level, characters can also be treated as weights. In the following, characters and weights are used without differences.

Assume $T$ acts on a space $X$ freely. $L(X / T, \lambda)=X \times_{T} \mathbb{C}_{\lambda}$ is a line bundle over $X / T$. The first Chern class of this linear bundle makes a connection between the group $\hat{T}$ of characters of $T$ and the second cohomology of $X / T$ :

$$
c_{1}: \hat{T} \rightarrow H^{2}(X / T), \quad \lambda \mapsto c_{1}(L(X / T, \lambda)) .
$$

Extend it to $\operatorname{Sym}(\hat{T})$ and $H^{*}(X / T)$, we get:

$$
c_{X / T}: \operatorname{Sym}(\hat{T}) \rightarrow H^{*}(X / T) .
$$

This map is called the characteristic map of $X / T$.

We take a special case as an example. If $X=G, X / T=G / T$. Then the generators $x_{i}$ of $H^{*}(G / T)$ is actually the image of the fundamental character $\lambda_{i}$ under the characteristic map $c_{G / T}$. By abusing the notations, $H^{*}(G / T)=\mathbb{Q}\left[x_{1}, \ldots, x_{n}\right] /\left(R_{+}^{W}\right)$, where $W$ is the Weyl group of $T$ in $G$ and $\left(R_{+}^{W}\right)$ is the ideal in $\mathbb{Q}\left[x_{1}, \ldots, x_{n}\right]$ generated by all homogeneous $W$-invariant elements of positive degree.

3.2. Equivariant cohomology. Let $G$ be a compact connected Lie group acting on a space $X$. Set $X_{G}:=E G \times_{G} X$. The equivariant cohomology $H_{G}^{*}(X)$ is defined to be $H^{*}\left(X_{G}\right)$. Note that $E G \times_{G} X \rightarrow B G$ is a fibre bundle over the classifying space $B G$ with the fibre $X$. If a torus $T$ acts on a point, $E T \times_{T} p t=B T$. Then $H_{T}^{*}(p t)=\mathbb{Q}\left[u_{1}, \ldots, u_{n}\right]$, where $\left\{u_{i}\right\}_{i=1}^{n}$ are the first Chern classes of the line bundles $L\left(B T, \lambda_{i}\right)$ described in the previous section.

Here does we describe another example, $H_{T}^{*}(G / T)$, which is essential in this paper. Since $(G / T)_{T}$ is a bundle over $B T$ with $G / T$ as its fibre, we have an embedding $i: G / T \hookrightarrow(G / T)_{T} . L_{\lambda}:=L(G / T, \lambda)=G \times_{T} \mathbb{C}_{\lambda}$ is a bundle over $G / T$. There is a natural left $T$-action on $L_{\lambda}$. Thus $\left(L_{\lambda}\right)_{T}$ is well-defined. It is easy to see that $\left(L_{\lambda}\right)_{T}$ is a line bundle over $(G / T)_{T}$ and $i^{*}\left(L_{\lambda}\right)_{T}=L_{\lambda_{i}}$. Then $i^{*} c_{1}\left(L_{\lambda_{i}}\right)_{T}=c_{1}\left(L_{\lambda_{i}}\right)=x_{i}$. In general, for an embedding $i: X \rightarrow X_{G}$, we say that an equivariant cohomology class $\tilde{\eta} \in H_{G}^{*}(X)$ is an equivariant extension of $\eta \in H^{*}(X)$ if $i^{*} \tilde{\eta}=\eta$. Thus $i^{*} c_{1}\left(L_{\lambda_{i}}\right)_{T}$ is an equivariant extension of $x_{i}$. We denote it by $\tilde{x}_{i}$.

Proposition 3.1. [4]. $H_{T}^{*}(G / T) \simeq \mathbb{Q}\left[u_{1}, \ldots, u_{n}, \tilde{x_{1}}, \ldots, \tilde{x_{n}}\right] / \mathcal{J}$, where $\mathcal{J}$ is the ideal generated by $b(\tilde{x})-b(u)$ for all homogeneous polynomials $b$ of positive degree invariant under the Weyl group action.

The following proposition is also very important.

Proposition 3.2. [4]. $H^{*}(X / G)$ is the subspace of $W$-invariants of $H^{*}(X / T)$. 
Remark 3.3. Combining the above two propositions, $H_{G}^{*}(G / T)$ is the $W$-invariants of $H_{T}^{*}(G / T)$. Thus $H_{G}^{*}(G / T) \simeq \mathbb{Q}\left[\tilde{x}_{1}, \ldots, \tilde{x}_{n}\right]$. Furthermore, $H_{G}^{*}(G / T) \simeq H_{T}^{*}(p t)$ naturally. The isomorphism is given by $\tilde{x}_{i} \leftrightarrow u_{i}$.

Similarly, for any closed Lie subgroup $H$ of $G$ containing $T$, we have

Proposition 3.4. (1) $H^{*}(X / H)$ is the subspace of $W_{H}$-invariants of $H^{*}(X / T)$, where $W_{H}$ is the Weyl group of $T$ in $H$.

(2) $H_{T}^{*}(G / H) \simeq \mathbb{Q}\left[u_{1}, \ldots, u_{n}\right] \otimes_{\mathbb{Q}}\left(\mathbb{Q}\left[\tilde{x_{1}}, \ldots, \tilde{x}_{n}\right]\right)^{W_{H}} / \mathcal{J}$, where $\mathcal{J}$ is the ideal generated by $b(\tilde{x})-b(u)$ for all homogeneous polynomials $b$ of positive degree invariant under the Weyl group action.

(3) $H_{G}^{*}(G / H) \simeq H_{H}^{*}(p t)$. The isomorphism is given by $\tilde{x}_{i} \leftrightarrow u_{i}$.

3.3. Equivariant Euler class. For a $G$-equivariant vector bundle $E \rightarrow X, E_{G} \rightarrow$ $X_{G}$ is also a vector bundle. The equivariant Chern class $c^{G}(E)$ of $E \rightarrow X$ is defined to be the ordinary Chern class of $E_{G} \rightarrow X_{G}$.

Let $V$ be a representation of $T$ with a weight decomposition $V=\oplus \mathbb{C}_{\lambda} . V \rightarrow$ $p t$ can be treated as an equivariant vector bundle over a point. Its equivariant homotopy is $V_{T} \rightarrow B T$, which is a vector bundle over $B T$. From the construction of $V_{T}$, it is very easy to see that $V_{T}=\oplus L(B T, \lambda)$. Thus the Chern class of the vector bundle $V_{T}$ is $\prod\left(1+u_{\lambda}\right)$, and the Euler class of this bundle is $\prod u_{\lambda}$. In other words, the equivariant Chern class of the equivariant vector bundle $V \rightarrow p t$ is $\prod\left(1+u_{\lambda}\right)$, and the equivariant Euler class $e^{T}$ is $\prod u_{\lambda}$. In this case to find the $T$-equivariant Euler class of $V \rightarrow p t$ is the same as that to find out the weight decomposition of $V$.

\section{The product formula}

4.1. Setup. We consider the equivariant cohomology of the $M_{\gamma}$ with $G_{\gamma}$-action. We use the standard model

$$
\operatorname{Gr}\left(d, \mathbb{C}^{\infty}\right):=\lim _{\rightarrow} \operatorname{Gr}\left(d, \mathbb{C}^{N}\right), N \rightarrow+\infty
$$

of the classifying space of $G L_{d}(\mathbb{C})$ for $d \geq 0$, and define

$$
B G_{\gamma}:=\prod_{i \in I} B G L\left(\gamma^{i}, \mathbb{C}\right)=\prod_{i \in I} G r\left(\gamma^{i}, \mathbb{C}^{\infty}\right) .
$$

Stack $\left[M_{\gamma} / G_{\gamma}\right]$ gives the universal family over $B G_{\gamma}$

$$
\left(M_{\gamma}\right)_{G_{\gamma}}:=E G_{\gamma} \times_{G_{\gamma}} M_{\gamma},
$$

where $E G_{\gamma} \rightarrow B G_{\gamma}$ is the standard universal $G_{\gamma}$-bundle.

Apply the propositions stated in the previous section, we have: 
Proposition 4.1. (1) $H_{G_{\gamma}}^{*}\left(M_{\gamma}\right) \simeq H_{G_{\gamma}}^{*}(p t)$ is an algebra of polynomials, with generators $\left\{u_{i, 1}, \ldots, u_{i, \gamma^{i}}\right\}_{i \in I}$, symmetric in the set of generators

$$
\left\{u_{i, 1}, \ldots, u_{i, \gamma^{i}}\right\}
$$

for each $i \in I$.

(2) $H_{G_{\gamma_{1}, \gamma_{2}}}^{*}\left(M_{\gamma_{1}, \gamma_{2}}\right) \simeq H_{G_{\gamma_{1}, \gamma_{2}}}^{*}$ (pt) is an algebra of polynomials, with generators $\left\{u_{i, 1}^{\prime}, \ldots, u_{i, \gamma_{1}^{i}}^{\prime}\right\}_{i \in I}$ and $\left\{u_{i, 1}^{\prime \prime}, \ldots, u_{i, \gamma_{2}^{i}}^{\prime \prime}\right\}_{i \in I}$, symmetric in these two sets respectively for each $i \in I$.

(3) $H_{G_{\gamma}}^{*}\left(G_{\gamma} / G_{\gamma_{1}, \gamma_{2}}\right)$ is an algebra of polynomials, with generators $\left\{\tilde{x_{i, 1}^{\prime}}, \ldots\right.$,

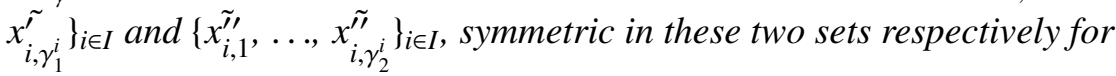
each $i \in I$. We have an isomorphism

$$
H_{G_{\gamma}}^{*}\left(G_{\gamma} / G_{\gamma_{1}, \gamma_{2}}\right) \simeq H_{G_{\gamma_{1}, \gamma_{2}}^{*}}^{*}(p t),
$$

and the isomorphism is given by $\tilde{x_{i, \alpha}} \leftrightarrow u_{i, \alpha}$.

Remark 4.2. There is a small gap here. The groups we are using in Section 3 along with many computations below are compact connected Lie groups, while the groups in our initial model are complex general linear groups, which is not compact. It does not really matter due to the following reason. Let $G$ be a compact connected Lie group, $T$ be a maximal torus, $G_{\mathbb{C}}$ be the complexification of $G$ and $B$ be the Borel subgroup containing $T$. By Iwasawa decomposition, $G_{\mathbb{C}} / B$ is $G$-equivariant diffeomorphic to $G / T$. Therefore when we want to compute the cohomology of the quotient space of a complex general linear group, we first construct one of its compact real forms and make quotients, apply all the theorems to the cohomology of these quotients, and then change back to the complex case to get the answer via the above diffeomorphism. Since we always use this trick, the procedure will not be repeated in the following and groups from either side are used freely.

4.2. Pullback. $M_{\gamma_{1}}$ and $M_{\gamma_{2}}$ could be treated as collections of $\gamma_{s}^{i} \times \gamma_{s}^{j}$ matrices where $s=1,2$ and $i, j \in I$, and $M_{\gamma_{1}, \gamma_{2}}$ can be realized as the space of block upper triangular matrices such that the upper left blocks are matrices from $M_{\gamma_{1}}$ and the lower right blocks from $M_{\gamma_{2}}$. Then $M_{\gamma_{1}, \gamma_{2}}$ is a subspace of $M_{\gamma}$ and $M_{\gamma_{1}}$ could be treated as a subspace of $M_{\gamma_{1}, \gamma_{2}}$. Similarly, $G_{\gamma_{1}, \gamma_{2}}$ can be treated as the subgroup of $G_{\gamma}$ which could preserve $M_{\gamma_{1}}$.

Lemma 4.3. $M_{\gamma_{1}, \gamma_{2}}, M_{\gamma_{1}} \times M_{\gamma_{2}}$ and $M_{\gamma}$ are equivariant homotopy equivalent to a point for any $\gamma_{1}, \gamma_{2}$, and $G_{\gamma_{1}, \gamma_{2}}$ is equivariant homotopy equivalent to $G_{\gamma_{1}} \times G_{\gamma_{2}}$.

Following the lemma, we have

$$
H_{G_{\gamma_{1}}}\left(M_{\gamma_{1}}\right) \otimes H_{G_{\gamma_{2}}}\left(M_{\gamma_{2}}\right) \cong H_{G_{\gamma_{1}} \times G_{\gamma_{2}}}\left(M_{\gamma_{1}} \times M_{\gamma_{2}}\right) \cong H_{G_{\gamma_{1}, \gamma_{2}}}\left(M_{\gamma_{1}, \gamma_{2}}\right) .
$$


Thus if at the beginning we have two cohomological classes $f\left(\left(u_{i, \alpha}^{\prime}\right)\right) \in H_{G_{\gamma_{1}}}^{*}\left(M_{\gamma_{1}}\right)$ and $g\left(\left(u_{i, \alpha}^{\prime \prime}\right)\right) \in H_{G_{\gamma_{2}}}^{*}\left(M_{\gamma_{2}}\right)$, after this pullback and multiplication in $H_{G_{\gamma_{1}, \gamma_{2}}^{*}}^{*}\left(M_{\gamma_{1}, \gamma_{2}}\right)$, we have $f\left(\left(u_{i, \alpha}^{\prime}\right)\right) g\left(\left(u_{i, \alpha}^{\prime \prime}\right)\right) \in H_{G_{\gamma_{1}, \gamma_{2}}}^{*}\left(M_{\gamma_{1}, \gamma_{2}}\right)$.

4.3. Pushforward in general. Given a proper $f: X \rightarrow Y$, the pushforward $f_{*}: H^{*}(X) \rightarrow H^{*}(Y)$ is defined in the following way. In two special cases the pushforword can be defined explicitly. If $f$ is an embedding, $f_{*}$ is to multiply the Euler class of the normal bundle of this embedding. If $f$ is a projection, $f: X \rightarrow Y$ can be treated as a bundle, and $f_{*}$ is defined to be the integration over fibers. Then if $f$ is an arbitrary proper map, we can decompose it into a composition of an embedding $i$ and a projection $\pi$, that is $f=\pi \circ i$, and thus we have $f_{*}=\pi_{*} \circ i_{*}$.

In our case, $p_{*}: H_{G_{\gamma_{1}, \gamma_{2}}}^{*}\left(M_{\gamma_{1}, \gamma_{2}}\right) \rightarrow H_{G_{\gamma}}^{*}\left(M_{\gamma}\right)$ is studied. We decompose the map $E G_{\gamma_{1}, \gamma_{2}} \times_{G_{\gamma_{1}, \gamma_{2}}} M_{\gamma_{1}, \gamma_{2}} \rightarrow E G_{\gamma} \times_{G_{\gamma}} M_{\gamma}$ into two steps. The first is $E G_{\gamma_{1}, \gamma_{2}} \times_{G_{\gamma_{1}, \gamma_{2}}} M_{\gamma_{1}, \gamma_{2}} \rightarrow E G_{\gamma_{1}, \gamma_{2}} \times_{G_{\gamma_{1}, \gamma_{2}}} M_{\gamma}$ which is an embedding, and the second is $E G_{\gamma_{1}, \gamma_{2}} \times_{G_{\gamma_{1}, \gamma_{2}}} M_{\gamma} \rightarrow E G_{\gamma} \times_{G_{\gamma}} M_{\gamma}$, which is a projection. Thus the pushforward can be decomposed into the following two steps:

$$
H_{G_{\gamma_{1}, \gamma_{2}}}\left(M_{\gamma_{1}, \gamma_{2}}\right) \rightarrow H_{G_{\gamma_{1}, \gamma_{2}}}\left(M_{\gamma}\right)
$$

and

$$
H^{*}\left(E G_{\gamma_{1}, \gamma_{2}} \times_{G_{\gamma_{1}, \gamma_{2}}} M_{\gamma}\right) \rightarrow H^{*}\left(E G_{\gamma} \times_{G_{\gamma}} M_{\gamma}\right) .
$$

In addition, since $M_{\gamma_{1}, \gamma_{2}}$ and $M_{\gamma}$ are equaivariant contractible, (4.6) can also be written

$$
H_{G_{\gamma}}^{*}\left(G_{\gamma} / G_{\gamma_{1}, \gamma_{2}}\right) \mapsto H_{G_{\gamma}}^{*}(p t) .
$$

Thus what we are going to do next is to study the equivariant Euler class of the normal bundle of the embedding $M_{\gamma_{1}, \gamma_{2}} \hookrightarrow M_{\gamma}$ to compute the first pushforward (4.5) and the $G_{\gamma}$-equivariant integration over Grassmannian $G_{\gamma} / G_{\gamma_{1}, \gamma_{2}}$ to compute the second pushforward (4.6).

Remark 4.4. In the computations of the following sections, what we would deal with is $G$-equivariant cohomology or $H$-equivariant cohomology rather than $T$ equivariant cohomology. However, most theorems stated above or below only work for $T$-equivariant cohomology, especially the localization formula. To solve this problem, we just treat a $G$-equivariant cohomological class as a $T$-equivariant cohomological class since $H_{G}^{*}(X)$ is a subset of $H_{T}^{*}(X)$. Then after using all the theorems, we obtain a $T$-equivariant cohomological class which is still $W$ invariant. It implies that it is actually a $G$-equivariant cohomological class. Therefore in the following parts, when applying theorems, we are always using $T$ equivariant cohomology. This allows the theorem to work, but makes no differences to the results.

\subsection{The first pushforward (4.5).}


4.4.1. The normal bundle of the embedding. $M_{\gamma}$ can be realized as a vector space of a collections of matrices, and $M_{\gamma_{1}, \gamma_{2}}$ is its subspace which is consisting of upper triangular matrices. Then the normal bundle of the embedding should be the product of $M_{\gamma_{1}, \gamma_{2}}$ and the normal subspace of $M_{\gamma_{1}, \gamma_{2}}$ with respect to $M_{\gamma}$, which is the subspace $N_{\gamma_{1}, \gamma_{2}}$ of left lower corner of matrices. Since the normal bundle is a product bundle, it implies that to find out the equivariant Euler class of normal bundle is to find the weight decomposition of $N_{\gamma_{1}, \gamma_{2}}$ with respect to $T \subset G_{\gamma_{1}, \gamma_{2}}$. Since $T$ acts on $M_{\gamma}$ and $M_{\gamma_{1}, \gamma_{2}}$ by conjugation, we have:

$$
N_{\gamma_{1}, \gamma_{2}}=\bigoplus_{a: i \rightarrow j} \bigoplus_{\alpha=1}^{\gamma_{1}^{j}} \bigoplus_{\beta=1}^{\gamma_{2}^{i}} \mathbb{C}_{\lambda_{i, \beta}^{\prime \prime}-\lambda_{j, \alpha}^{\prime}} .
$$

Then we know the equivariant Euler class is

$$
e^{T}\left(N_{\gamma_{1}, \gamma_{2}}\right)=\prod_{a: i \rightarrow j} \prod_{\alpha=1}^{\gamma_{1}^{j}} \prod_{\beta=1}^{\gamma_{2}^{i}}\left(u_{i, \beta}^{\prime \prime}-u_{j, \alpha}^{\prime}\right)
$$

4.4.2. Computations. We start from $f\left(\left(u_{i, \alpha}^{\prime}\right)\right) g\left(\left(u_{i, \alpha}^{\prime \prime}\right)\right) \in H_{G_{\gamma_{1}, \gamma_{2}}}^{*}\left(M_{\gamma_{1}, \gamma_{2}}\right)$. After the first pushforward, we get

$$
f\left(\left(u_{i, \alpha}^{\prime}\right)\right) g\left(\left(u_{i, \alpha}^{\prime \prime}\right)\right) \prod_{a: i \rightarrow j} \prod_{\alpha=1}^{\gamma_{1}^{j}} \prod_{\beta=1}^{\gamma_{2}^{i}}\left(u_{i, \beta}^{\prime \prime}-u_{j, \alpha}^{\prime}\right) .
$$

4.5. The second pushforward (4.6). We are going to use Localization formula to compute this pushforward.

4.5.1. Localization formula for $T$-equivariant cohomology. Let $T$ act on $X$, with $X_{F}$ being the set of fixed points. The equivariant cohomology of $X$ can be gotten from the equivariant cohomology of the set of fixed points $X_{F}$. This is called the equivariant localization theorem. See [1] for details. To integrate the cohomology $\eta$ over $X$, or to consider the pushforward of the projection $\pi: X \rightarrow p t$, a localization formula is obtained from the theorem:

$$
\pi_{*}(\eta)=\sum_{Z} i_{Z *} \frac{i_{Z}^{*} \eta}{e^{T}\left(N_{Z}\right)},
$$

where $Z$ is a connected component of $X_{F}, i_{Z}$ is the embedding of this component into $X$, and $N_{Z}$ is the normal bundle of the embedding.

In the case that all the fixed points are isolated, the normal bundle $N_{p}$ of the embedding of the fixed point $p$ is the tangent space $T_{p}$ at $p$. Hence the equivariant 
localization formula can be expressed as

$$
\pi_{*}(\eta)=\sum_{p \in X_{F}} i_{p_{*}} \frac{i_{p}^{*} \eta}{e^{T}\left(T_{p}\right)} .
$$

Therefore we need to know how to compute the pullback of classes to fixed points and how to compute the equivariant Euler classes associated to the fixed points.

4.5.2. Pullback of some classes. $G / T$ is a $T$-space with left multiplication action. The set of fixed points is labeled by the element in the Weyl group $W_{G}$. Let $\omega \in W_{G}$. Recall that $u_{i}$ and $\tilde{x}_{i}$ be generators of $H_{T}^{*}(G / T)$.

Proposition 4.5. [4].The pullback of equivariant cohomology from $G / T$ to its fixed point $\omega$ is described as below:

(1) $i_{\omega}^{*}\left(u_{i}\right)=u_{i}$;

(2) $i_{\omega}^{*} \tilde{x}_{i}=\omega \cdot u_{i}=u_{\omega(i)}$.

4.5.3. The equivariant Euler class associated to the fixed points. We consider the tangent space at the fixed point $\omega \in W_{G}$. For the Grassmannian $G_{\gamma} / G_{\gamma_{1}, \gamma_{2}}$, the tangent space is isomorphic to $\prod_{i \in I} \operatorname{Hom}\left(\mathbb{C}^{\gamma_{1}^{i}}, \mathbb{C}^{\gamma^{i}} / \mathbb{C}^{\gamma_{1}^{i}}\right)$, and the weight decomposition of the tangent space at the fixed point indexed by $1 \in W$ is

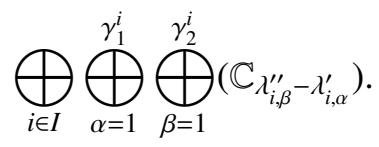

Thus the equivariant Euler class is

$$
\prod_{i \in I} \prod_{\alpha=1}^{\gamma_{1}^{i}} \prod_{\beta=1}^{\gamma_{2}^{i}}\left(u_{i, \beta}^{\prime \prime}-u_{i, \alpha}^{\prime}\right)
$$

For the tangent space at the point indexed by $\omega \in W$, we only need to change the order of weights, and get

$$
e^{T}\left(T_{\omega}\right)=\prod_{i \in I} \prod_{\alpha=1}^{\gamma_{1}^{i}} \prod_{\beta=1}^{\gamma_{2}^{i}}\left(u_{i, \omega(\beta)}^{\prime \prime}-u_{i, \omega(\alpha)}^{\prime}\right) .
$$

4.5.4. Computations. We start from $f\left(\left(u_{i, \alpha}^{\prime}\right)\right) g\left(\left(u_{i, \alpha}^{\prime \prime}\right)\right) \prod_{a: i \rightarrow j} \prod_{\alpha=1}^{\gamma_{1}^{j}} \prod_{\beta=1}^{\gamma_{2}^{i}}\left(u_{i, \beta}^{\prime \prime}-u_{j, \alpha}^{\prime}\right)$. First by using Proposition 4.1, we transfer the cohomological class from $H_{T}^{*}(p t)$ to $H_{G}^{*}(G / T)$ and get

$$
f\left(\left(x_{i, \alpha}^{\tilde{\prime}}\right)\right) g\left(\left(\tilde{x_{i, \alpha}^{\prime \prime}}\right)\right) \prod_{a: i \rightarrow j} \prod_{\alpha=1}^{\gamma_{1}^{j}} \prod_{\beta=1}^{\gamma_{2}^{i}}\left(x_{i, \beta}^{\tilde{\prime}}-{\tilde{x_{j, \alpha}^{\prime}}}^{\tilde{y}}\right) .
$$


At the fixed point indexed by $\omega \in W_{G_{\gamma_{1}, \gamma_{2}}}$, the equivariant Euler class of the embedding of $\omega$ is

$$
\prod_{i \in I} \prod_{\alpha=1}^{\gamma_{1}^{i}} \prod_{\beta=1}^{\gamma_{2}^{i}}\left(u_{i, \omega(\beta)}^{\prime \prime}-u_{i, \omega(\alpha)}^{\prime}\right)
$$

and

$$
i_{\omega}^{*} f\left(\left(\tilde{x_{i, \alpha}}\right)\right)=f\left(\left(u_{i, \omega(\alpha)}\right)\right) .
$$

Thus the result of the pushforward is

$$
\begin{aligned}
& \pi_{*}\left(f\left(\left(x_{i, \alpha}^{\tilde{\prime}}\right)\right) g\left(\left(x_{i, \alpha}^{\tilde{\prime}}\right)\right) \prod_{a: i \rightarrow j} \prod_{\alpha=1}^{\gamma_{1}^{j}} \prod_{\beta=1}^{\gamma_{2}^{i}}\left(\tilde{x}_{i, \beta}^{\tilde{\prime}}-\tilde{x}_{j, \alpha}^{\tilde{r}}\right)\right) \\
= & \sum_{\omega} \frac{f\left(\left(u_{i, \omega(\alpha)}^{\prime}\right)\right) g\left(\left(u_{i, \omega(\alpha)}^{\prime \prime}\right)\right) \prod_{a: i \rightarrow j} \prod_{\alpha=1}^{\gamma_{1}^{j}} \prod_{\beta=1}^{\gamma_{2}^{i}}\left(u_{i, \omega(\beta)}^{\prime \prime}-u_{j, \omega(\alpha)}^{\prime}\right)}{\prod_{i \in I} \prod_{\alpha=1}^{\gamma_{1}^{i}} \prod_{\beta=1}^{\gamma_{2}^{i}}\left(u_{i, \omega(\beta)}^{\prime \prime}-u_{i, \omega(\alpha)}^{\prime}\right)} .
\end{aligned}
$$

Since the Weyl group in this case is the set of all shuffles, we can reinterpret the above result as a shuffle product. Also if we let $a_{i j}$ be the number of arrows in $Q$ from vertex $i$ to $j, \prod_{a: i \rightarrow j}\left(u_{i, \beta}^{\prime \prime}-u_{j, \alpha}^{\prime}\right)=\prod_{i, j \in I}\left(u_{i, \beta}^{\prime \prime}-u_{j, \alpha}^{\prime}\right)^{a_{i j}}$. Then we come to the following formula:

Theorem 4.6. [3] The product $f \cdot g$ of elements $f \in H_{\gamma_{1}}$ and $g \in H_{\gamma_{2}}$ is given by the symmetric function $h\left(\left(u_{i, \alpha}\right)_{i \in I, \alpha \in\left\{1, \ldots, \gamma^{i}\right\}}\right)$, where $\gamma=\gamma_{1}+\gamma_{2}$, obtained from the following function in variables $\left(u_{i, \alpha}^{\prime}\right)_{i \in I, \alpha \in\left\{1, \ldots, \gamma_{1}^{i}\right\}}$ and $\left(u_{i, \alpha}^{\prime \prime}\right)_{i \in I, \alpha \in\left\{1, \ldots, \gamma_{2}^{i}\right\}}$,

$$
f\left(\left(u_{i, \alpha}^{\prime}\right)\right) g\left(\left(u_{i, \alpha}^{\prime \prime}\right)\right) \frac{\prod_{i, j \in I} \prod_{\alpha=1}^{\gamma_{1}^{j}} \prod_{\beta=1}^{\gamma_{2}^{i}}\left(u_{i, \beta}^{\prime \prime}-u_{j, \alpha}^{\prime}\right)^{a_{i j}}}{\prod_{i \in I} \prod_{\alpha=1}^{\gamma_{1}^{i}} \prod_{\beta=1}^{\gamma_{2}^{i}}\left(u_{i, \beta}^{\prime \prime}-u_{i, \alpha}^{\prime}\right)},
$$

by taking the sum over all $\prod_{i \in I}\left(\begin{array}{l}\gamma^{i} \\ \gamma_{1}^{i}\end{array}\right)$ shuffles for any given $i \in I$ of the variables $u_{i, \alpha}^{\prime}$ and $u_{i, \alpha}^{\prime \prime}$.

Acknowledgement. I thank to Yan Soibelman who introduced me to this subject and taught me a lot. I also thank to Zongzhu Lin, Zhaobin Fan, Janu Verma and Jie Ren for useful conversations and communications.

\section{References}

[1] Atiyah, M.F. and Bott, R. The moment map and equivariant cohomology. Topology, 1984. 23(1): 1-28

[2] Behrend, K. Cohomology of stacks. In Intersection theory and moduli, ICTP Lect. Notes, XIX. Abdus Salam Int. Cent. Theoret. Phys., Trieste, 2004. 249-294 (electronic) 
[3] Kontsevich, M. and Soibelman, Y. Cohomological hall algebra, exponential hodge structures and motivic Donaldson-Thomas invariants. ArXiv e-prints, 2010

[4] Tu, L.W. Computing characteristic numbers using fixed points. In A celebration of the mathematical legacy of Raoul Bott, volume 50 of CRM Proc. Lecture Notes. Amer. Math. Soc., Providence, RI, 2010. 185-206 